\title{
New approach for a "point-like" neutron source creation based on sharp focusing of a high quality deuteron beam produced by high-current gasdynamic ECR ion source
}

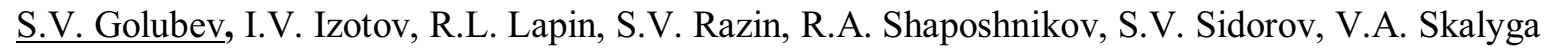 \\ Institute of Applied Physics of Russian academy of Sciences, Nizhny Novgorod, Russia, gol@appl.sci-nnov.ru
}

\section{Introduction}

Neutron tomography is one of the most exciting recent achievements of nuclear physics. It is of note that neutron tomography requires dedicated neutron source, i.e. paraxial sources with low angle spread. The only sources now able to deliver required neutron beams with sufficient intensity are nuclear reactors and large-scale accelerators in pair with collimators [1, 2]. Collimation degree is usually lies in the range of 50-300 (ratio between length and width), and the neutron flux density at collimator's end is $10^{8}-10^{9} \mathrm{~s}^{-1} \mathrm{~cm}^{-2}$ as a maximum.

The use of point-like neutron sources based on laser plasma induced by focusing of powerful femtosecond laser radiation onto a neutron-producing target [3, 4], for example set of deuterium clusters was discussed lately. Ions are effectively accelerated at certain conditions in such plasma, producing neutrons as a result of D-D collisions. The neutron yield of $10^{6}$ per $1 \mathrm{~J}$ of laser energy has been detected at the source size of $100 \mu \mathrm{m}[3,4]$. However state-of-the-art lasers having $0.1 \mathrm{~J}$ per pulse at $1 \mathrm{kHz}$ repetition rate give average neutron yield of $10^{8} \mathrm{~s}^{-1}$.

The possibility of a point-like neutron source creation with the yield of $10^{10}-10^{13} \mathrm{~s}^{-1}$ based on high-current ion source of a new generation was discussed in [5]. It was proposed to use either D-D or D-T reactions occurring at the bombardment of D or T-loaded target with focused D ion beam. A size of the neutron emitting area would be determined by the quality of the ion beam and the efficiency of the beam focusing system (ie, the minimum size of the bombardment area). This proposal is based on recent developments [6-8] of the unique high-current ion source. Such source utilize high-power gyrotron millimeter-wave radiation for plasma production in an open magnetic trap under conditions of electron cyclotron resonance (ECR). In recent experiments it was demonstrated that this source with simple two-electrode extraction system and only $45 \mathrm{kV}$ maximum voltage on it is able to produce deuterium ion beams with $400 \mathrm{~mA}$ current and normalized rms emittance less than $0.1 \pi \cdot \mathrm{mm} \cdot \mathrm{mrad}$. Bombardment of a heavy ice target with described beam provided a total neutron yield of the order of $10^{9} \mathrm{~s}^{-1}$.

Present paper describes first experiments on proton beam focusing dedicated to the point-like neutron source development based on high current ECR ion source.

\section{Experimental setup}

Experimental studies of ion beam properties and search for optimal discharge conditions for high brightness beam formation were carried out at SMIS 37 experimental facility [6-8]. SMIS 37 provides gyrotron radiation at $37.5 \mathrm{GHz}$ frequency with peak power up to $100 \mathrm{~kW}$ in $1.5 \mathrm{~ms}$ pulses for plasma heating. Microwaves were focused in the center of a discharge chamber $25 \mathrm{~cm}$ long and $4 \mathrm{~cm}$ in diameter by means of special quasioptical microwave-to-plasma coupling system. The discharge chamber was placed in a magnetic trap of simple mirror configuration. The magnetic field in the trap was produced by means of pulsed solenoids, spaced $15 \mathrm{~cm}$ apart. Magnetic field in the mirror was varied from 1.4 to $4 \mathrm{~T}$ (ECR field for $37.5 \mathrm{GHz}$ is $1.34 \mathrm{~T}$ ). Ratio of the maximum and minimum magnetic fields of the trap was equal to 5 (i.e. $\mathrm{B}_{\max } / \mathrm{B}_{\min }$, mirror ratio). Deuterium or hydrogen were used as a working gas. The gas inlet into the source was realized through a gas feed line incorporated with the microwave coupling system. The ion extraction and beam formation were realized by two-electrode, i.e. single gap plasma electrode - puller electrode system. Holes in plasma electrode and puller were $5 \mathrm{~mm}$ and $10 \mathrm{~mm}$ respectively. The plasma electrode was placed as far as $10 \mathrm{~cm}$ downstream from the magnetic mirror to limit the extracted ion flux, which helps improving the beam transport through the puller. The maximum available extraction voltage was $50 \mathrm{kV}$. A Faraday cup was placed right behind the puller electrode to measure the total beam current passing through the extractor. Emittance of the extracted beam (all species together) was measured with "pepper-pot" method, which has been successfully tested earlier at SMIS 37 [6-8].

In the first experiment on beam focusing a simple magnetic lens with $2 \mathrm{~T}$ maximum field on the axis was used. It was installed just after the puller electrode. Beam current distribution and its focusing efficiency was measured using a scintillator plate for beam visualization in the focal plane of the lens.

\section{Experimental results}

The dependence of the ion beam current measured with the Faraday cup on the acceleration voltage is shown in Fig. 1 for the optimal extraction electrode configuration.

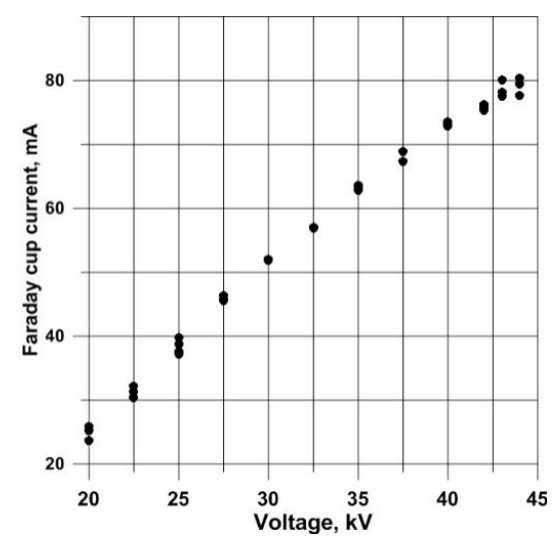

Fig. 1. Faraday cup current versus extraction voltage 
For beam emittance measurement "pepper-pot" plate was placed $1 \mathrm{~cm}$ downstream from the puller with another $5.5 \mathrm{~cm}$ gap before a CsI scintillator for beam imaging. Example of the reconstructed emittance diagram is shown in fig. 2. The RMS normalized emittance appeared to be $0.06 \pi \cdot \mathrm{mm} \cdot \mathrm{mrad}$.

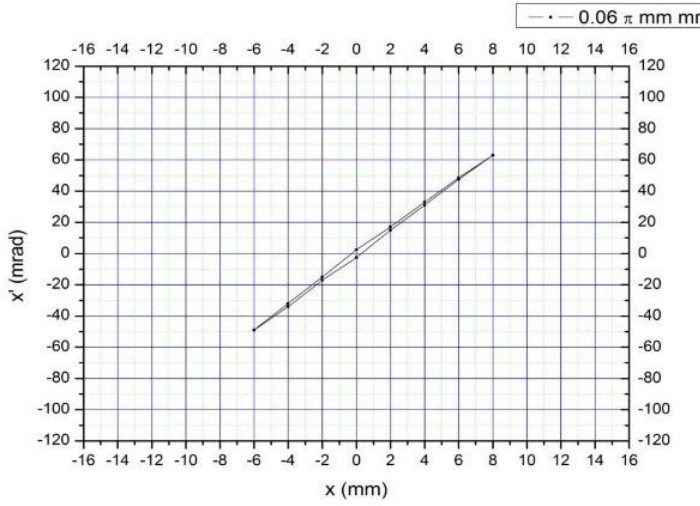

Fig. 2. Ion beam emittance diagram. Extractor: $5 \mathrm{~mm}$ plasma electrode aperture and $10 \mathrm{~mm}$ puller aperture with $11 \mathrm{~mm}$ gap. $\mathrm{rms}$ normalized emittance is be $0.06 \pi \cdot \mathrm{mm} \cdot \mathrm{mrad}$
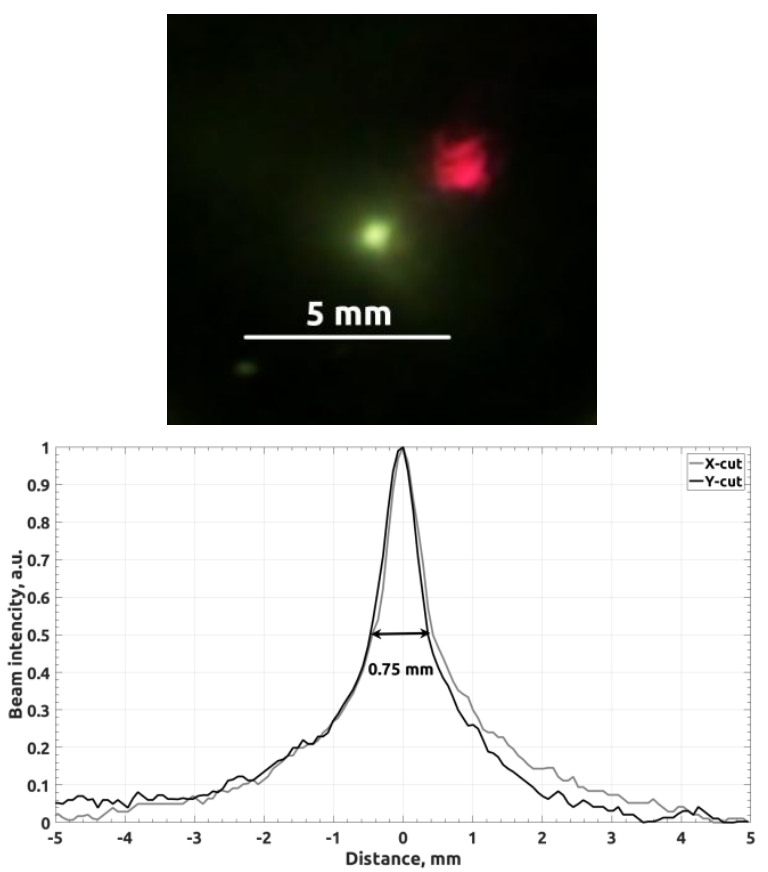

Fig. 3. Upper image: photograph of scintillator luminescence (green glow) induced by $80 \mathrm{~mA}$ hydrogen beam in magnetic lens focal plane (red glow corresponds to optical emission from plasma through the plasma electrode aperture); Lower image: corresponding beam space distribution in two orthogonal radial directions
Thus produced ion beam with $80 \mathrm{~mA}$ current and $0.06 \pi \cdot \mathrm{mm} \cdot \mathrm{mrad}$ emittance had the normalized $\mathrm{rms}$ brightness $22 \mathrm{~A} /(\pi \cdot \mathrm{mm} \cdot \mathrm{mrad})^{2}$.

As it was mentioned above the magnetic lens was used for beam focusing as the next step. Measurements have shown that lens focus for $1.3 \mathrm{~T}$ field was situated approximately in $10 \mathrm{~cm}$ from lens center. Example of scintillator luminescence image induced by $45 \mathrm{keV}$, $80 \mathrm{~mA}$ hydrogen beam together with its analysis of space distribution in radial direction are presented in fig. 3.The best result for focused beam full width at half maximum was $0.75 \mathrm{~mm}$.

\section{Conclusion}

The use of a high-current ECR ion source with quasigasdynamic plasma confinement and heating with gyrotron microwave radiation allows the formation of light ion beams with uniquely low emittance (for a given current level). Such a low emittance enables the focusing of the ion beam into the small spot. In frames of suggested approach to development of a point-like neutron source at the first experimental step-beam size at the focus of the magnetic lens less than $1 \mathrm{~mm}$ was demonstrated. Estimation presented in [5] show that the point-like source based on high current ECR ion source could produce the neutron yield up to $10^{11} \mathrm{~s}^{-1}$ for D-target, and up to $10^{13} \mathrm{~s}^{-1}$ for T-target. Such neutron flux corresponds to a flux density of $10^{8}-10^{10} \mathrm{~s}^{-1} \mathrm{~cm}^{-2}$ at $10 \mathrm{~cm}$ distance from the source, which may be effectively utilized for neutron tomography, thus making this technique widely available.

\section{Acknowledgments}

Presented work was supported by the grant of Russian Science Foundation \# 16-19-10501.

\section{References}

1. Viktor L. Aksenov, Physics-Uspekhi, V.40,N. 5(1997).

2. Ian S. Anderson, Robert L. McGreevy, Hassina Z. Bilheux. Neutron Imaging and Applications. Springer (2009).

3.T. Ditmire, et.al. Physics of Plasmas v.7, p.1993 (2000).

4. J. Davis, G. M. Petrov, and A. L. Velikovich. Physics of Plasmas 13, 064501 (2006).

5. S. Golubev, V. Skalyga, I. Izotov, A. Sidorov.Journal of Instrumentation, v. 12, 2017 JINST_12_T02003 (2017)

6. V. Skalyga, et. al.JINST, v.7, P10010 (2012).

7. V. Skalyga, et. al. Review of Scientific Instruments, v. 85 , no. 2, p. 02A702-1 - 02A702-3 (2014).

8. V. Skalyga, I. et.al. Journal of Applied Physics v.118, p. 093301 (2015). 